\title{
Substituição de núcleo metálico fundido por pino de fibra de vidro anatomizado: relato de caso
}

Replacement of metallic core fused by anatomized fiberglass pin: case report

Reemplazo del core metálico usado por el pin de fibra anatomizado: informe del caso

Aiane Maria Ferreira CONRADO ${ }^{1}$

Euriliana Anísia de Moura Marcelino FERREIRA ${ }^{\mathbf{1}}$

Bruno Amorim Santos de ALBUQUERQUE ${ }^{2}$

Ana Luisa Cassiano Alves BEZERRA ${ }^{3}$

Rodivan BRAZ ${ }^{4}$

Márcia de Almeida DURÃO 5

${ }^{1}$ Curso de Graduacão Odontologia do Centro Universitário Mauricio de Nassau - UNINASSAU/ PE, 52011-000, Recife/PE, Brasil

${ }^{2}$ Mestre em Dentística, Faculdade de Odontologia, Universidade de Pernambuco- FOP/UPE

${ }^{3}$ Mestranda em Clínica Odontológica, Faculdade de Odontologia, Universidade de Pernambuco- FOP/UPE, 50.100-010, Camaragibe/PE, Brasil ${ }^{4}$ Doutor em Dentística, Professor Adjunto da Faculdade de Odontologia, Universidade de Pernambuco- FOP/UPE, 50.100-010, Camaragibe/PE, Brasil ${ }^{5}$ Pós-Doutoranda em Dentística, Faculdade de Odontologia, Universidade de Pernambuco- FOP/UPE, 50.100-010, Camaragibe/PE, Brasil; Docente do Curso de Odontologia, Centro Universitário Mauricio de Nassau - UNINASSAU/ PE, 52011-000, Recife/PE, Brasil

\section{Resumo}

Dentes tratados endodonticamente, com canais amplos e grande perda de remanescente são de difícil reabilitação, tornandose grande desafio para cirurgiões dentistas. Esses casos frequentemente requerem utilização de pinos para restabelecimento funcional e estético. Este trabalho teve como objetivo relatar um caso clínico de substituição do pino metálico por pino de fibra de vidro personalizado com resina composta. Paciente, sexo feminino, compareceu a uma clínica odontológica privada na cidade do Recife-PE, ao exame clinico e radiográfico apresentava incisivo central superior direito, com tratamento endodôntico insatisfatório e núcleo metálico fundido. Foi indicado a substituição por um pino de fibra de vidro anatomizado com resina composta. Realizou-se retratamento endodôntico. O pino de fibra foi desinfetado com álcool $70 \%$, preparado com Silano, sistema adesivo e fotopolimerização. A resina composta foi depositada sobre o pino, levado ao conduto, fazendo movimentos de inserção e remoção, copiando o formato intrarradicular. O cimento resinoso autoadesivo dual foi utilizado e porção coronária reconstruída. O uso do pino de fibra de vidro mostrou-se uma excelente opção para o tratamento reabilitador de dentes fragilizados, absorvendo melhor cargas mastigatórias, maior adaptação as paredes do canal, além da longevidade da reabilitação restauradora.

Descritores: Pinos Dentários; Resinas Compostas; Reabilitação; Técnica para Retentor Intrarradicular.

\section{Abstract}

Teeth that are endodontically treated, with wide canals and great residual loss are hard to rehabilitate, becoming a great challenge for dental surgeons. These cases frequently require the use of pins for functional and aesthetic restoration. This project has as objective to report a clinical case of the replacement of a metallic pin, by a fiberglass pin costumed with composite resin. A female patient reported to a private dental clinic in Recife-PE, where in the clinical and radiographic exam, the right upper central incisor presented unsatisfactory endodontic treatment and a metal post restoration. It was suggested to be replaced by a fiberglass pin anatomized by composite resin. Endodontic treatment was performed. The fiberglass pin was disinfected with $70 \%$ alcohol, prepared with silane, dental adhesive and photopolymerization. The composite resin was inserted on the pin, taken to the conduit, with movements of removal and insertion, copying the intra-root format. The dual self-adhesive resinous cement was utilized and a portion of the crown was reconstructed. The use of the fiberglass pin proved to be a good option for rehabilitation treatment on fragile teeth, absorbing better masticatory charges, better adaptation to the root walls, as well as the restorative rehabilitation longevity.

Descriptors: Dental Pins; Composite Resins; Rehabilitation; Post and Core Technique.

\section{Resumen}

Los dientes tratados endodónticamente, con canales anchos y gran pérdida de remanente son difíciles de rehabilitar, por lo que es un gran desafío para los dentistas. Estos casos a menudo requieren el uso de pines para la restauración funcional y estética. Este estudio tenía como objetivo informar de un caso clínico de sustitución del pasador de metal por un pasador de fibra de vidrio personalizado con resina compuesta. Una paciente asistió a una clínica dental privada en la ciudad de Recife-PE, en un examen clínico y radiográfico, con un incisivo central derecho temprano, con un tratamiento de endodoncia insatisfactorio y un núcleo metálico fundido. El reemplazo fue indicado por un pin de fibra de vidrio anatomizado con resina compuesta. Se realizó un retratamiento de endodoncia. El pasador de fibra fue desinfectado con $70 \%$ de alcohol, preparado con Silane, sistema adhesivo y curado ligero. La resina compuesta fue depositada en el pasador, llevada al conducto, realizando movimientos de inserción y eliminación, copiando la forma intraradicular. Se utilizó doble cemento de resina autoadhesiva y se reconstruyó la porción coronaria. El uso del pasador de fibra de vidrio resultó ser una excelente opción para el tratamiento de rehabilitación de dientes frágiles, absorbiendo mejores cargas masticatorias, mayor adaptación a las paredes del canal, además de la longevidad de la rehabilitación restaurativa.

Descriptores: Pines Dentales; Resinas Compuestas; Rehabilitación; Técnica de Perno Muñón.

INTRODUÇÃO

Dentes com tratamentos endodônticos, cáries extensas e destruição de coroa clínica apresentam maior risco à fratura da estrutura dentária. A utilização de retentores intrarradicular é uma técnica onde elementos com grande perda estrutural são reabilitados por meio de uma fixação intra-canal, promovendo uma melhor estética, maior durabilidade e função ${ }^{1,2}$.

Durante muito tempo, os núcleos

metálicos fundidos foram utilizados como única opção para este tipo de tratamento reabilitador. Porém, o custo laboratorial, longo tempo de trabalho, tensões que podem ocasionar fratura radicular, possibilidade de manchamento pela corrosão e sua elevada rigidez ${ }^{3}$, contribuíram para o desenvolvimento dos pinos de fibra de vidro $^{4}$. Além de apresentarem propriedades físicas semelhantes à dentina, minimizam o risco de fraturas, favorecem à estética ${ }^{5}$ e 
apresentam execução simplificada, sendo imprescindível que o clínico tenha conhecimento e habilidade acerca de suas indicações e técnicas adequadas ${ }^{3}$.

A técnica direta é mais simples e rápida, devendo o diâmetro do conduto radicular ser compatível ao diâmetro do pino, prevendo mínimo desgaste, evitando o enfraquecimento da estrutura dentária ${ }^{6}$. Entretanto, dentes com amplos condutos, os pinos pré-fabricados podem não ter uma boa adaptação, necessitando de uma camada mais espessa de cimento, o que pode promover maior estresse ao remanescente e baixa resistência de união do conjunto ${ }^{7}$. Assim, torna-se necessário lançar mão de outras técnicas para a uma melhor anatomização e adaptação do pino ao conduto radicular.

O objetivo deste estudo foi descrever através de um relato de caso, a substituição de um núcleo metálico fundido por um pino de fibra de vidro pré-fabricado anatomizado com resina composta.

\section{CASO CLÍNICO}

Paciente do sexo feminino, 33 anos, procurou por atendimento odontológico especializado, na cidade do Recife/PE, com queixa de dor em dente anterior, incisivo central superior direito, relatando ter feito tratamento endodôntico e uma coroa cerâmica. Após ser esclarecida sobre o conteúdo do estudo e o tratamento planejado, foi solicitada a assinatura do TCLE - Termo de Consentimento Livre e Esclarecido, e a autorização para uso de sua imagem. Em seguida, foi realizado o exame clínico e radiográfico onde foi constatado uma lesão na região periapical, resultado do selamento apical insatisfatório e a presença de um núcleo metálico fundido (Figura 1).

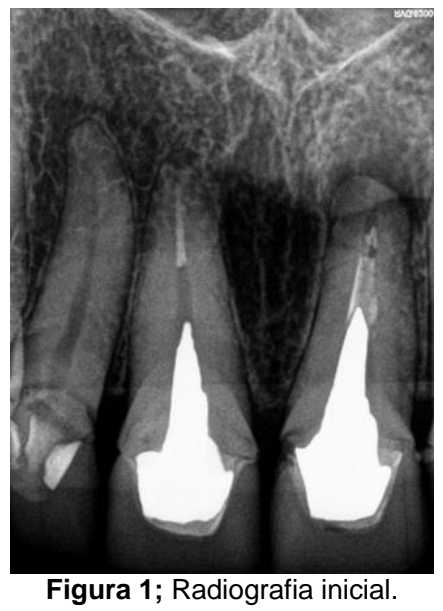

Como tratamento, foi indicado 0 retratamento do canal e a substituição do núcleo metálico, pelo pino de fibra de vidro. Por se tratar de um dente anterior e com um amplo canal, optou-se pela técnica semidireta, anatomizando o pino com resina composta para sua melhor adaptação ao conduto radicular. A remoção da coroa foi desgastada com ponta diamantada esférica $n^{\circ} 1015$ (KG Sorensen, São Paulo, Brasil) (Figura 2A), em seguida o núcleo metálico foi cortado lateralmente com uma broca carbide transmetal de ponta arredondada (Predator Transmetal, Prima Dental, Reino Unido) (Figura 2B). Com auxílio de vibrações ultrassônicas, houve 0 descolamento da estrutura metálica do conduto radicular (Figura $2 \mathrm{C}$ ), promovendo sua remoção (Figura 2D) sem causar prejuízos à estrutura dental.
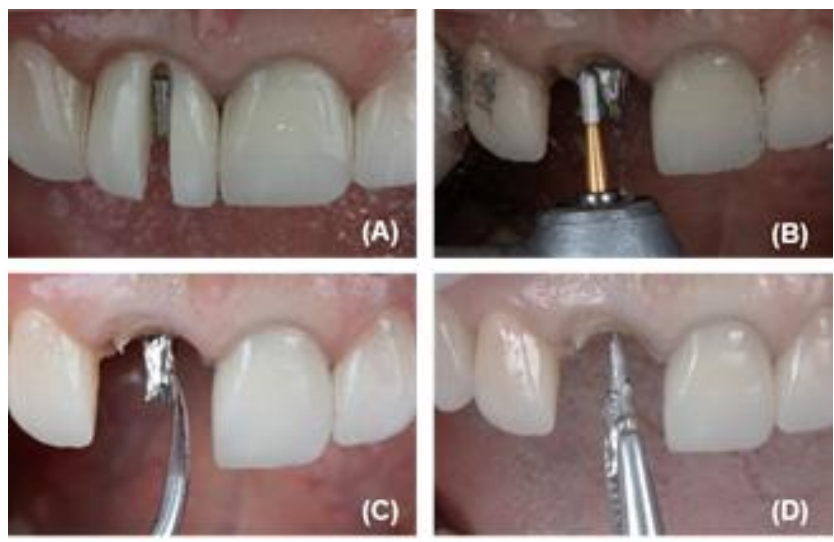

Figura 2: Desgaste da coroa com ponta diamantada $(A)$, corte lateral do núcleo metálico $(B)$, descolamento da estrutura metálica do conduto radicular com uso de ultrassom (C) e remoção do núcleo metálico fundido (D).

Para o retratamento endodôntico, foi instalado o isolamento absoluto com lençol de borracha (Nic Tone, Romênia), grampo 212 (Duflex, SS White, Brasil), e selamento com a barreira gengival (Top Dam, FGM, Brasil).

Após uma semana, tempo para cura final do cimento obturador, foi realizada desobstrução deixando-se $4 \mathrm{~mm}$ de selamento apical com guta percha aquém do comprimento de trabalho (Figura 3A). Procedeu-se a limpeza do conduto radicular por meio de irrigação com água destilada (blister), seguida de aspiração e secagem com cones de papel absorvente. A partir da odontometria, selecionou-se o pino de fibra de vidro 0,5 (White Post DC, FGM, Brasil), este foi introduzido no conduto para marcação de seu comprimento (Figura 3B), em seguida o conduto foi lubrificado gel hidrossolúvel (K-Y gel, Johnson \& Johnson, EUA) (Figura 3C).

A limpeza e desinfecção do pino de fibra de vidro foi realizada com fricção de uma gaze embebida em álcool $70 \%$, na sequência o silano (Monobond N, Ivoclar Vivadent, Liechtenstein), foi aplicado com microbrush, deixando-o agir por 60 segundos e breve forte jato de ar (Figura 4A). Em seguida, foi aplicado o sistema adesivo (Tetric N-Bond Universal, Ivoclar Vivadent, 
Liechtenstein) por 20 segundos (Figura 4B) e fotopolimerizado com Bluephase $\mathrm{N}$ (Ivoclar Vivadent, Liechtenstein), com intensidade de $1.200 \mathrm{~mW} / \mathrm{cm}^{2}$, por 10 segundos, seguindo instruções do fabricante(Figura 4C).
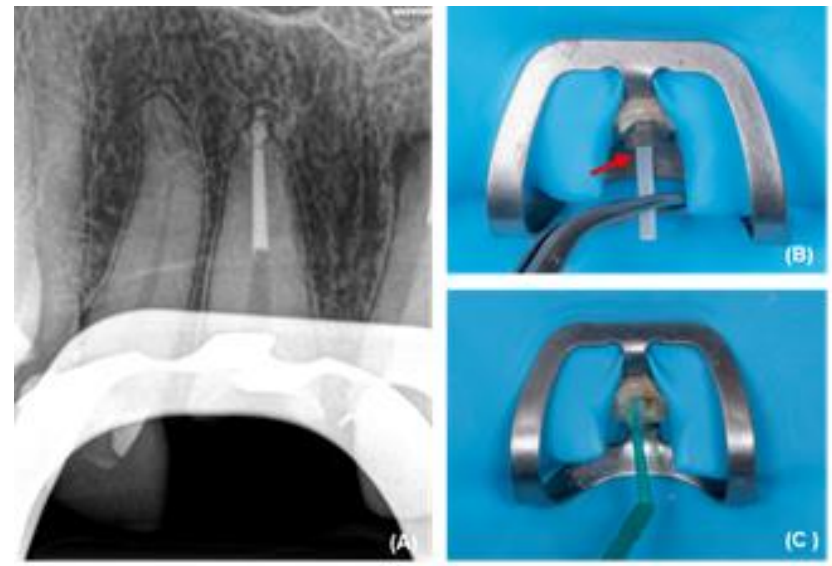

Figura 3: Radiografia da desobturação do tratamento endodôntico, preservando $4 \mathrm{~mm}$ do selamento apical (A), prova do pino de fibra de vidro 0,5 (White Post DC, FGM, Brasil) (B) e lubrificação do conduto radicular com gel hidrossolúvel (C).
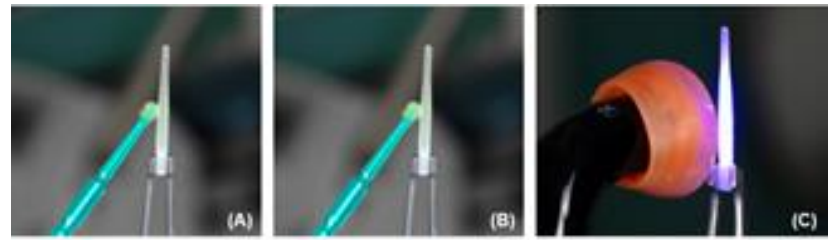

Figura 4: Aplicação do silano sobre o pino, com auxílio de um microbrush, que foi seguido de uma espera de 60 s e secando com breve e forte jato de ar (A), aplicação do sistema adesivo por 20 segundos $(B)$ e fotopolimerizado por 10 segundos $(C)$.

Um incremento de resina composta convencional (Empress Direct, Ivoclar Vivadent, Liechtenstein) foi colocado em torno do pino(Figura 5A), em seguida este foi inserido no conduto para sua modelagem(Figura 5B), tendo-se o cuidado de demarcar a face vestibular como referência. Sendo feita uma breve fotopolimerização, posicionando a luz em direção ao longo eixo do dente por 5 segundos (Figura 5C), em seguida, faz-se movimentos repetidos de remoção e inserção do pino no conduto, sendo então, reposicionando, pressionando e fotopolimerizado por mais 5 segundos, repetindo esta sequência por mais duas vezes completando 0 ciclo de fotopolimerização total de 20 segundos.
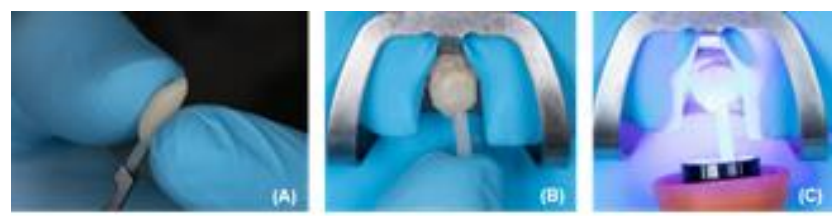

Figura 5: Inserção de resina composta convencional em torno do pino (A), inserção do pino junto com a resina no conduto para sua modelagem $(B)$ e fotoativação com luz direcionada ao longo eixo do dente, por $5 \mathrm{~s}(\mathrm{C})$.

Após esse tempo, o pino foi removido do conduto e fotopolimerizado por mais 40 segundos (Figura 6A e 6B), garantindo total fotopolimerização da resina. Para remoção do gel hidrossolúvel, usou-se álcool $70 \%$ no pino anatomizado (Figura 6C), e o conduto foi irrigado com blister de água destilada (Figura 6D), aspirando com sugador endodôntico e secagem com cone de papel absorvente.
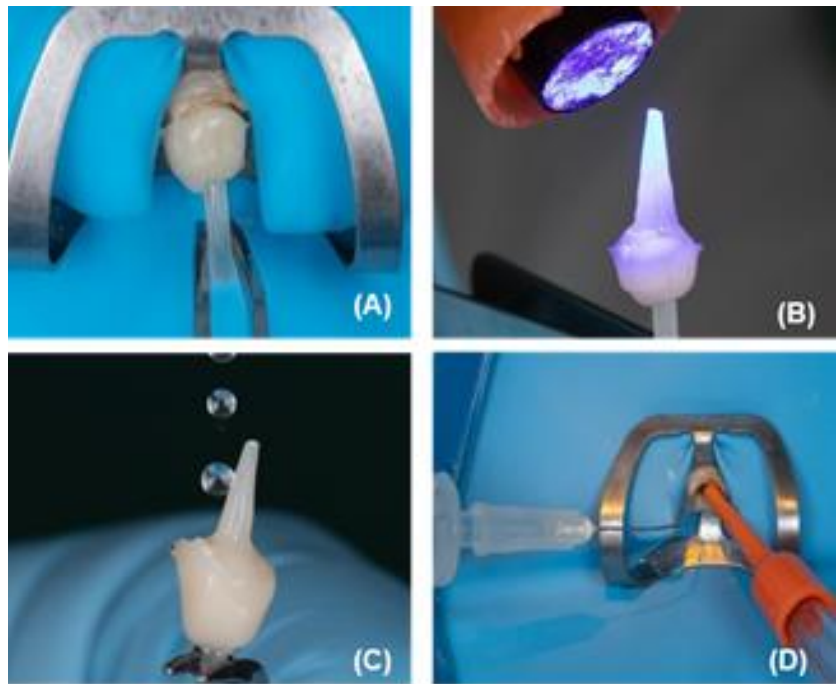

Figura 6: Remoção do pino do conduto (A), seguido de fotoativação por mais 40 s(B). Remoção do gel hidrossolúvel do pino com Álcool $70 \%$ (C) e irrigação do conduto com blister de água destilada (D)

A cimentação foi realizada com cimento resinoso autoadesivo dual (RelyX U200, 3M ESPE, EUA), na cor A2, dispensando tratamento prévio do remanescente dental. Foram utilizadas duas medidas para mistura (Figura 7A), misturado manualmente (Figura 7B), adaptado em uma seringa e ejetado cuidadosamente da porção apical para cervical (Figura 7C E 7D), visando evitar a formação de bolhas. O pino já anatomizado foi inserido e pressionado no conduto respeitando seu correto posicionamento (Figura 7E), os excessos de cimento foram removidos com o auxílio de microbrush, e realizada a fotopolimerização final por 20 segundos.

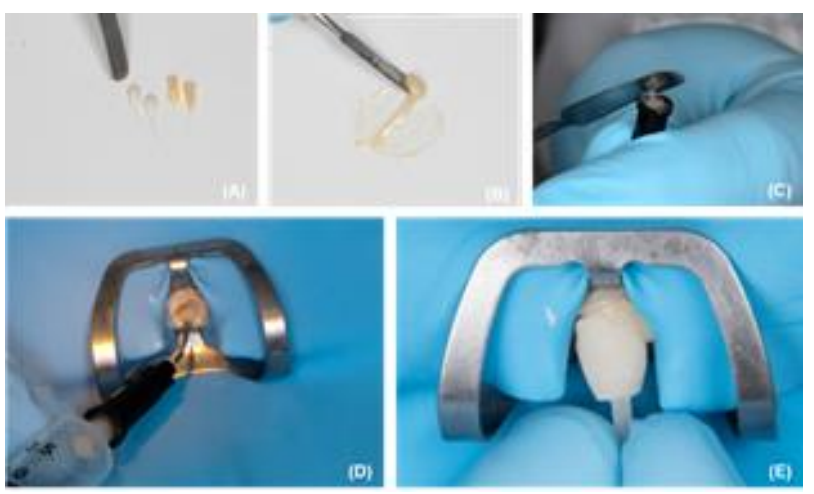

Figura 7: Medidas do cimento resinoso (A), mistura manual (B), adaptação em uma seringa (C), inserção dentro do conduto radicular $(D)$ e posicionamento do pino anatômico no canal $(E)$.

Após a cimentação, o isolamento absoluto foi removido e feito o desgaste do pino com ponta diamantada, tendo como referência o plano incisal. A porção coronária foi preparada com ponta diamantada tronco-cônica de ponta 
arredondada 856 (Komet, Alemanha) (Figura $8 \mathrm{~A})$, permitindo término cervical e angulação adequada do núcleo na porção coronária e reabilitação com coroa provisória para posterior reabilitação com coroa definitiva (Figura 8B e $8 \mathrm{C})$.

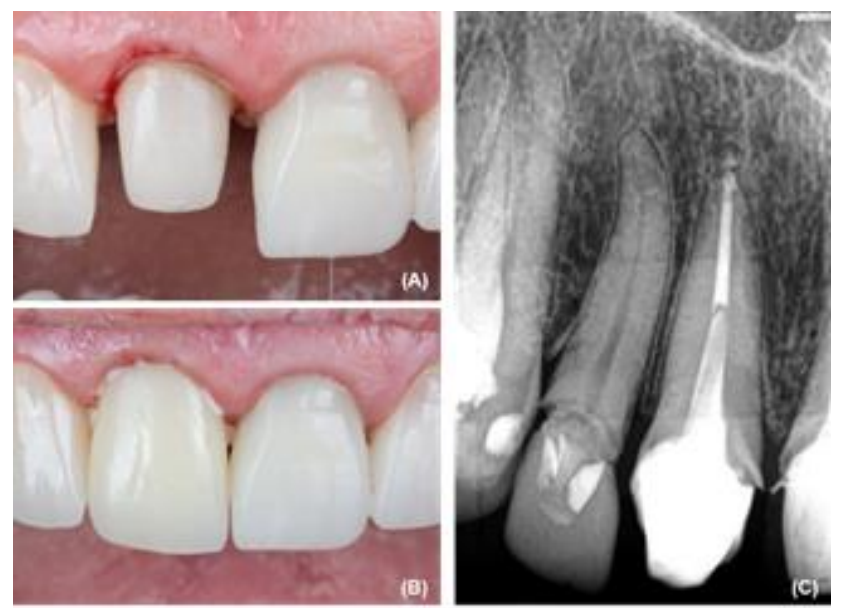

Figura 8: Finalização do preparo da porção coronária $(A)$, cimentação da coroa provisória (B) e radiografia final do dente (C).

DISCUSSÃO

Restaurações de dentes endodonticamente tratados sempre foram casos desafiadores na Odontologia. Devido à perda de substâncias orgânicas, 0 dente perde a elasticidade em sua composição, tornando-se susceptível a fraturas devido a sua rigidez estrutural ${ }^{8}$. Filter et al. $^{9}$ evidenciaram que em alguns casos, há a necessidade de um retentor intrarradicular, principalmente em dentes com grande perda de estrutura coronária, explicado pela necessidade de ter uma retenção da coroa a ser confeccionada, e não somente para dar reforço ao remanescente dental.

Por muito tempo, os núcleos metálicos fundidos foram utilizados como reforço em dentes com tratamento de canal. No entanto, suas desvantagens foram favorecendo 0 desenvolvimento de outros materiais, surgindo assim o pino de fibra de vidro ou pinos préfabricados $^{10}$. A partir de vários estudos sobre 0 uso de retentores intrarradiculares, tornou-se possível o entendimento sobre a alternativa de substituição de um núcleo metálico fundido por um pino de fibra de vidro e sua anatomização com resina composta. Os pinos de fibra de vidro ganharam muito espaço no âmbito odontológico, por não sofrerem corrosão, promover preparo conservador, apresentarem menor tempo clínico e excelente estética, quando comparado ao núcleo metálico fundido, permitindo que seu uso seja realizado através de diversas técnicas ${ }^{2,5,7}$.

A técnica utilizada neste caso, semidireta ou também chamada de anatomização, se dá pela realização da cópia negativa do conduto, através da moldagem intrarradicular com resina composta depositada sobre o pino de fibra de vidro, obtendo o formato perfeito do conduto e a reconstrução da porção coronária ${ }^{11}$. Essa anatomização favorece uma maior adaptação as paredes do canal e a distribuição uniforme do agente cimentante, garantindo maior retenção e adaptação, principalmente em canais amplos ${ }^{7}$, como os canais dos incisivos centrais superiores.

Assim como neste estudo, Filho et al. ${ }^{5}$ demostraram que para dentes com amplos canais, é indicada a anatomização do pino de fibra reduzindo a pressão gerada na interface e aumentando a resistência as forças mastigatórias, corroborando com Fantin ${ }^{4}$ que observou que, apesar das grandes chances de falhas relacionadas as inúmeras etapas que compõem a técnica de anatomização, ainda é uma excelente alternativa favorecendo uma melhor retenção do pino.

Uma outra alternativa para dentes com amplos canais são os pinos de fibra de vidro personalizados e fresados através de CAD/CAM. Para Libonati et al. ${ }^{12}$ a técnica permite que, através de um escaneamento digital, produzam-se pinos de vibra de vidro anatômicos, totalmente adaptados ao conduto radicular, melhorando a biomecânica do dente tratado endodonticamente, a adesão às paredes do conduto e reduzindo a incidência de fraturas.

Corrêa et al. ${ }^{13}$ realizaram uma revisão sistemática e meta-analises de estudos in vitro que avaliavam os valores de resistência à fratura e modo de falha de raízes fragilizadas restauradas com núcleos metálicos fundidos, pinos de fibra de vidro e pinos anatômicos. Em seus resultados, observaram que ao analisar os modos de falhas, os estudos evidenciaram maiores números de fraturas desfavoráveis para os grupos restaurados com núcleos metálicos fundidos, podendo ser explicadas pelas propriedades mecânicas do material. Concluíram também que os menores valores de resistência apresentaram-se nos pinos de fibra de vidro, no entanto a fraturas ocasionadas eram do tipo favorável, preservando a estrutura radicular.

Quanto a escolha de cimentação para este caso, ao optar por utilizar o sistema de cimento resinoso autoadesivo, eliminam-se etapas que podem gerar falhas de cimentação e comprometer a longevidade do tratamento reabilitador. Para Marques et al. $^{14}$, que observaram a resistência a tração por teste push-out de pinos de fibra de vidro cimentados através de três estratégias adesivas (sistema adesivo convencional simplificado + cimento 
resinoso dual, sistema adesivo convencional de três passos + cimento resinoso dual e cimento autoadesivo), não houve alteração significativa entre os tipos de técnicas, apesar do resultado ser maior no grupo que utilizou o sistema adesivo convencional de três passos + cimento resinoso dual. Também, Sarkis-Onofre et al. ${ }^{15}$, ao avaliarem longevidade de pinos de fibra de vidro e metálicos fundidos comparando tipo de cimento, não encontraram diferenças significativas entre o cimento resinoso dual e o autoadesivo no caso dos pinos de fibra de vidro. Apesar dos pinos de fibra de vidro apresentarem propriedades mais favoráveis, as suas limitações, em alguns casos, pode favorecer o uso do núcleo metálico fundido, como é o caso de reabilitação de dentes com remanescente dental menor que $2 \mathrm{~mm}^{6}$. Logo, para um planejamento de um retentor intrarradicular, é imprescindível o conhecimento do profissional sobre técnicas que possam ser aplicadas, bem como, um correto diagnóstico, considerando também as limitações, vantagens e desvantagens que cada técnica oferece.

CONCLUSÃO

Foi possível concluir que os pinos de fibra de vidro anatomizados com resina composta são excelente opção para reabilitação de dentes tratados endodonticamente, restabelecendo sua função e estética. Principalmente em dentes fragilizados, com amplo conduto radicular, possibilitando uma espessura mais fina da linha de cimento.

\section{REFERÊNCIAS}

1. Zavanelli AC, Zavanelli RA, Mazaro JVQ, Lemos CAA, Dias ES, Silva EV et. at. Coroas Endoncrown: uma revisão de literatura e relato de caso. Arch Health Invest. 2017;6(8):382-89.

2. Santos-Filho PC, Veríssimo C, Raposo LH, Noritomi MecEng PY, Marcondes Martins LR Influence of Ferrule, Post System, and Length on Stress Distribution of Weakened Root-filled Teeth. J Endod. 2014;40(11):1874-78.

3. Pereira $\mathrm{HC}$, Feliciano JA, Nascimento $F$, Pereira VG, Viana HC - Aplicação Clínica de Pino de Fibra de Vidro: Relato de Caso. Revista de Odontologia Contemporânea ROC. 2017;1(2):60-1.

4. Fantin LL - Resistência push out de pinos anatômicos confeccionados com resina convencional e diferentes tipos de resinas bulk fill [dissertação]. Cuiabá; Programa de PósGraduação em Ciências Odontológicas Integradas, Universidade de Cuiabá; 2017.

5. Guimarães RC, Guimarães RI, Pennisi PRC, Prudente MS, Santos-Filho PCF, Martins VM. Pino de fibra de vidro reanatomizado com resina composta: um relato de caso. ROC; 2017;1(2):64-9.

6. Soares DNS, Sant'Ana LLP. Estudo comparativo entre pino de fibra de vidro e pino metálico fundido: uma revisão de literatura. Id on Line Rev Mult Psic. 2018;12(42):996-1005.

7. Miorando B, Vermudt A, Ghizoni JS, Pereira JR, Pamato S. Utilização de Pinos IntraRadiculares. Miorando et al. J Res Dent. 2018. 6(1):16-22.

8. SÁ TCM, Akaki E, Sa JCM - Pinos Estéticos: Qual o Melhor Sistema? Arq bras odontol. 2010;6(3):179-84.

9. Filter VP, Zanetti F, Simoneti R, Rocha GC, Tonial D, Durand LB. Restauração semidireta associada a um retentor intrarradicular em dente anterior. Dentística on line. 2011; 10(21):5.

10. Novais VR, Rodrigues RB, Simamoto Júnior PC, Lourenço CS, Soares CJ. Correlation between the mechanical properties and structural characteristics of different fiber posts systems. Braz Dent J. 2016;27(1):46-51.

11. Ferreira MBC, Carlini-Júnior $B$, Silva-Sousa YT, Gomes EA, Spazzin AO. Pino de fibra de vidro anatômico: relato de caso. J Oral Invest. 2018; 7(1);52-61.

12. Libonati A, Di Taranto V, Gallusi G, Montemurro E, Campanella V. CAD/CAM customized glass fiber post and core with digital intraoral impression: a case report. Clin Cosmet Invest Dent. 2020;12:17-24.

13. Corrêa G, Brondani LP, Onofre RS, Bergoli CD. Restorative strategies for weakened roots: Systematic reviw and Meta-analysis of in vitro studies. Braz Dent Sci. 2019;22(1):124-34.

14. Marques VF, Araújo ECF, Faria e Silva AL, Tapety CMC, Moreira MAG, Casselli DSM. Avaliação de resistência de união entre dentina e pinos de fibra de vidro utilizando três diferentes técnicas de cimentação. RFO. 2014; 19(3):283-87.

15. Sarkis-Onofre R, Jacinto RC, Boscato N, Cenci MS, Pereira-Cenci T. Cast metal vs. glass fibre posts: a randomized controlled trial with up to 3 years of follow up. J Dent. 2014;42(5):582-87. 


\section{CONFLITO DE INTERESSES}

Os autores declaram não haver conflitos de interesse

\section{AUTOR PARA CORRESPONDÊNCIA}

\section{Márcia de Almeida Durão}

Universidade de Pernambuco, Faculdade de

Odontologia de Pernambuco

Av. Gov. Agamenon Magalhães, s/n - Santo Amaro

50.100-010 Recife-PE, Brasil

E-mail: marciadurao21@gmail.com

Submetido em 08/05/2020

Aceito em 23/10/2020 